\title{
Dietary purslane (Portulaca oleracea L.) promotes the growth performance of broilers by modulation of gut microbiota
}

\author{
Cong Wang ${ }^{1}$, Qing Liu ${ }^{1,2}$, Fengchun Ye ${ }^{3}$, Hongbo Tang ${ }^{1}$, Yanpeng Xiong ${ }^{1}$, Yongfei Wu ${ }^{1}$, Luping Wang , \\ Xuanbiao Feng ${ }^{1}$, Shuiyin Zhang ${ }^{1,2}$, Yongmei Wan ${ }^{1}$ and Jianhua Huang ${ }^{1,2^{*}}$
}

\begin{abstract}
Purslane is a widespread wild vegetable with both medicinal and edible properties. It is highly appreciated for its high nutritional value and is also considered as a high-quality feed resource for livestock and poultry. In this study, Sanhuang broilers were used to investigate the effect of feeding purslane diets on the growth performance in broilers and their gut microbiota. A total of 48 birds with good growth and uniform weight were selected and randomly allocated to four treatment groups A (control), B, C and D. Dietary treatments were fed with basal diet without purslane and diets containing 1\%,2\% and 3\% purslane. The $16 \mathrm{~S}$ rDNA was amplified by PCR and sequenced using the Illumina HiSeq platform to analyze the composition and diversity of gut microbiota in the four sets of samples. The results showed that dietary inclusion of $2 \%$ and $3 \%$ purslane could significantly improve the growth performance and reduce the feed conversion ratio. Microbial diversity analysis indicated that the composition of gut microbiota of Sanhuang broilers mainly included Gallibacterium, Bacteroides and Escherichia-Shigella, etc. As the content of purslane was increased, the abundance of Lactobacillus increased significantly, and Escherichia-Shigella decreased. LEfSe analysis revealed that Bacteroides_caecigallinarum, Lachnospiraceae, Lactobacillales and Firmicutes had significant differences compared with the control group. PICRUSt analysis revealed bacteria mainly enriched in carbohydrate metabolism pathway due to the additon of purslane in the diet. These results suggest that the addition of purslane to feed could increase the abundance of Lactobacillus in intestine, modulate the environment of gut microbiota and promote the metabolism of carbohydrates to improve its growth performance. This study indicates that the effect of purslane on the growth-promoting performance of broilers might depend on its modulation on gut microbiota, so as to provide a certain scientific basis for the application of purslane in the feed industry.
\end{abstract}

Keywords: Purslane, Sanhuang broilers, Growth performance, Gut microbiota, Carbohydrate metabolism

\section{Introduction}

Sanhuang broiler chicken, also is known as triple-yellow chicken, is one of the most famous breeds of poultry as well as native chickens in China, named for its yellow beak, yellow feather and yellow claws. The meat of Sanhuang chicken is tender, delicious and nutritious, and contains high protein and amino acid content, but low

\footnotetext{
*Correspondence: huangjh1113@sina.com

2 Jiangxi Key Laboratory of Bioprocess Engineering, Jiangxi Science and Technology Normal University, 330013 Nanchang, China Full list of author information is available at the end of the article
}

fat content. Sanhuang chicken is also a great source of trace elements such as iron, copper and zinc, and is rich in vitamins, which can be used to nourish blood and keep healthy. In the livestock and poultry breeding industry, the performance of livestock and poultry is an important factor affecting economic benefits. However, slow growth is another characteristic of Chinese local breeds. Some studies have indicated that added purslane to the feed of broiler chickens has a good effect on enhanced the appetite of animals or promoted the growth performance (Habibian et al. 2019; Kartikasari et al. 2018). 
Purslane (Portulaca oleracea L.) is an annual herb that is widely distributed around the globe. Purslane is a nutritious vegetable with high antioxidant properties. It is rich in nutrients such as glucose and malic acid, and is also recognized as the richest source of $\alpha$-linolenic acid, essential omega- 3 fatty acids, ascorbic acid, $\alpha$-tocopherol and $\beta$-carotene (Uddin et al. 2014). Omega-3 polyunsaturated fatty acids (PUFAs) are essential nutrients for health benefits such as reducing the risk of coronary heart disease and depression, regulating metabolic syndrome, and relieving anxiety (Schunck et al. 2018; Shahidi and Ambigaipalan 2018). Purslane contains bioactive compounds like flavonoids, alkaloids, polysaccharides, etc., which have a variety of pharmacological effects, such as antibacterial, antioxidant, hypoglycemic, hypolipidemic, neuroprotection and so on (Rahimi et al. 2019; Zhou et al. 2015). The edible and medicinal value of purslane is of momentous significance to the growth and development of organisms, and it has shown great nutritional potential and has very far-reaching application prospects in the future.

The gut microbiota is essential for the health of the host, for example, to promote host metabolism, defense against pathogens, enhance immunity, etc., and directly or indirectly affect most of the host's physiological functions. Meanwhile, the intestinal flora is involved in metabolic diseases such as obesity and dyslipidemia of the organism. The interplay of the gut microbiota with host can be mediated by diet, and its effects starts but is far beyond the intestine (Dabke et al. 2019). At present, antibiotics are widely used in animal husbandry and weight gain by gut microbiota manipulation to improve the growth performance of animals (Angelakis 2017). Although they have specific benefits to the health of animals, some pathogens develop resistance to them quickly, and if the abuse of antibiotics in livestock feed may lead to the transfer of drug-resistant pathogens to humans. Therefore, it is necessary to develop new products as a substitute for antibiotics in feed to better promote the health and growth performance of poultry, which is also a major guarantee for the health of humans who consume such animal products.

Traditional Chinese herbal medicine contains numerous biologically active compounds, which can provide various nutritional and health benefits to animals, so it can be used as a feed additive (Abdallah et al. 2019). As a feed additive, purslane is especially worthy of attention in enhancing nutrition, regulating metabolism and promoting the digestion and absorption of nutrients in animals. This study aims to investigate the effect of purslane on the growth performance and gut microbiota in broiler chickens, as well as the role of these intestinal bacteria in regulating the growth and metabolism. In practical application, it is possible to achieve the purpose of partially replacing antibiotics and gaining weight, which provides a certain scientific basis for the application of purslane in animal feed industry.

\section{Materials and methods Feeding management and diets}

The experiment was carried out in a chicken farm in Jian City, Jiangxi Province, China, and it lasted for 30 days from November 5 to December 4, 2019. A total of 48 Sanhuang chickens with good growth and body weight of $285 \pm 30 \mathrm{~g}$ were selected and randomly allocated to four treatment groups A (control), B, C and D, with 3 replicates in each group and 4 chickens in each replicate. During the entire test period, the designated personnel took up the responsibility to manage tasks such as feeding, drinking and cleaning. Infrared lamp was used for heating, the temperature was maintained at $19-28{ }^{\circ} \mathrm{C}$, and normal ventilation and lighting were kept. Fed once in the morning and afternoon, and eat freely throughout the test period.

The dietary used were all powdered feeds, and the basic diet consisted mostly of corn and soybean meal. The detailed dietary and nutritional composition is presented in Additional file 1: Table S1. The A group was fed only the basal diet, and the diets of the $\mathrm{B}, \mathrm{C}$ and $\mathrm{D}$ groups were supplemented with $1 \%, 2 \%$ and $3 \%$ purslane, respectively. All of purslane were collected from the wild area and ground into powder after washed and dried for feeding trials.

\section{Sample collection, DNA extraction and amplification}

On the last day of the test, the final weight of each chicken was measured and three fecal samples were collected from each group. A total of twelve fecal samples were stored in different sterile cryotube and placed in liquid nitrogen, then brought back and transferred to the $-80{ }^{\circ} \mathrm{C}$ refrigerator for storage until further analysis.

Genomic DNA of all feces samples were extracted according to the specifications of kit. The concentration and purity of DNA were detected by NanoDrop 2000 (Thermo, USA), while the quality and integrity of DNA were detected by $1 \%$ agarose gel electrophoresis. The $\mathrm{V} 3+\mathrm{V} 4$ region of bacterial $16 \mathrm{~S}$ rDNA was amplified by PCR with the specific primers, where the primers were designed according to the conserved region, and then sequencing adapters were added to the end of primers for PCR amplification. PCR amplification was conducted under the following conditions: $5 \mathrm{~min}$ at $94{ }^{\circ} \mathrm{C}$ for initialization, $30 \mathrm{~s}$ denaturation at $94{ }^{\circ} \mathrm{C}$ for 30 cycles, $30 \mathrm{~s}$ annealing at $52{ }^{\circ} \mathrm{C}$, and $30 \mathrm{~s}$ extension at $72{ }^{\circ} \mathrm{C}$, and a final 10 min elongation at $72{ }^{\circ} \mathrm{C}$. 


\section{Sequencing and data processing}

After PCR amplification, the products were purified, quantified and homogenized to get a sequencing library. Then library quality control was performed for constructing libraries, qualified libraries were paired-end sequenced by Illumina HiSeq 2500 platform of Biomarker Technologies (Beijing, China). According to overlap relation between paired-end reads, FLASH software (v1.2.11) (Magoc and Salzberg 2011) was used to merge the paired reads into a sequence and to filter out nonconforming tags in order to collect the raw tags. The minimum overlap length was $10 \mathrm{bp}$ and the maximum error ratio was 0.2. Trimmomatic software (v0.33) (Bolger et al. 2014) was used to filter merged raw tags to get high quality clean tags. The sliding window was $50 \mathrm{bp}$, and the average quality in sliding window was 20. Finally, UCHIME software (v8.1) (Edgar et al. 2011) was used to identify and remove chimera sequences to get effective tags.

\section{Microbial community and diversity analysis}

The USEARCH software (v10.0) (Edgar 2013) was used to cluster all effective tags for all samples at $97 \%$ similarity level. In general, if the similarity between sequences is higher than $97 \%$, it can be defined as an operational taxonomic unit (OTU), and each OTU corresponds to a representative sequence. The OTU was filtered when species abundance less than $0.005 \%$ (Bokulich et al. 2013). Then the Ribosomal Database Project (RDP) classifier (Wang et al. 2007) was used to classify OTUs under the $80 \%$ threshold and perform taxonomic annotation for OTU on the basis of Silva (Bacteria) (Release132, http://www. arb-silva.de) (Quast et al. 2013) taxonomic database.

The QIIME software (v1.9.1) (Caporaso et al. 2010) was used to count the community composition of each sample and generate the species abundance table at different taxonomic levels, and then use $\mathrm{R}$ language tool to draw community structure graph of samples at different taxonomic levels. Furthermore, QIIME software was used to select OTU sequences with the highest abundance at genus taxonomic level as the representative sequence to perform multiple sequences alignment and phylogenetic tree construction, and then the graph was drawn through the Python language tool. With the phylogenetic relationship and relative abundance information of each OTU in the samples, the species annotation results of a single sample was visualized by KRONA software (Ondov et al. 2011). According to the taxonomic database of existing microbial species provided by NCBI, MEGAN5 software (Huson et al. 2007) was used to return the species abundance information obtained by sequencing to the taxonomic system relation tree of the database to understand the evolutionary relationships and abundance differences of all microorganisms in the samples. According to the OTU abundance table, Mothur software (v1.30) (Schloss et al. 2009) was used to evaluate the Alpha diversity and the Chaol and Shannon indices were calculated to compare the differences in species abundance and diversity between groups (Chao et al. 2006).

\section{LEfSe analysis and PICRUSt prediction}

The linear discriminant analysis (LDA) effect size (LEfSe) analyses were performed on the website http://hutte nhower.sph.harvard.edu/galaxy (Segata et al. 2011). According to the established Biomarker screening criteria (LDA score $>4$ ), the eligible biomarkers were identified. Functional capacity of gut microbiota was predicted using the Phylogenetic Investigation of Communities by Reconstruction of Unobserved States (PICRUSt) (Parks et al. 2014). First, the generated OTU-table needs to be standardized. Then, according to the unique greengene id corresponding to each OTU, the KEGG family information related to OTU can be obtained, so as to calculate the abundance of KEGG (Kyoto Encyclopedia of Genes and Genomes) and compare it with KEGG database to obtain functional predictions.

\section{Statistical analysis}

The statistical analysis of all recorded data in the experiment was carried out by $\mathrm{R}$ statistical software. One-way analysis of variance (ANOVA) was used for multiple samples, and the differences between groups were examined by two-tailed Student's t-test. The G-test and Fisher test methods in STAMP were used to test the significant differences in species abundance between different samples, and pairwise T-tests was applied between different groups. Test results of $\mathrm{P}<0.05$ were considered significant, and $\mathrm{P}<0.01$ were considered extremely significant.

\section{Results}

Effect of purslane on the growth performance of Sanhuang chickens

In this study, we randomly divided 48 Sanhuang chickens into 4 groups with 12 chickens in each group. To investigate the effect of purslane on growth performance, we recorded the initial and final weight of each chicken and measured the daily intake of each group. The four groups had no significant difference regarding initial weight.

After the end of the test period, we compared the average daily food intake and weight gain of each group, and the changes in feed conversion ratio (FCR) were analyzed (Fig. 1). There was no significant difference in the daily food intake between the four groups $(P>0.05)$, but the average daily weight gain of groups $C$ and D with $2 \%$ and $3 \%$ purslane was significantly higher than that of the control group A $(\mathrm{P}<0.05)$, which increased by $1.44 \mathrm{~g}$ and 

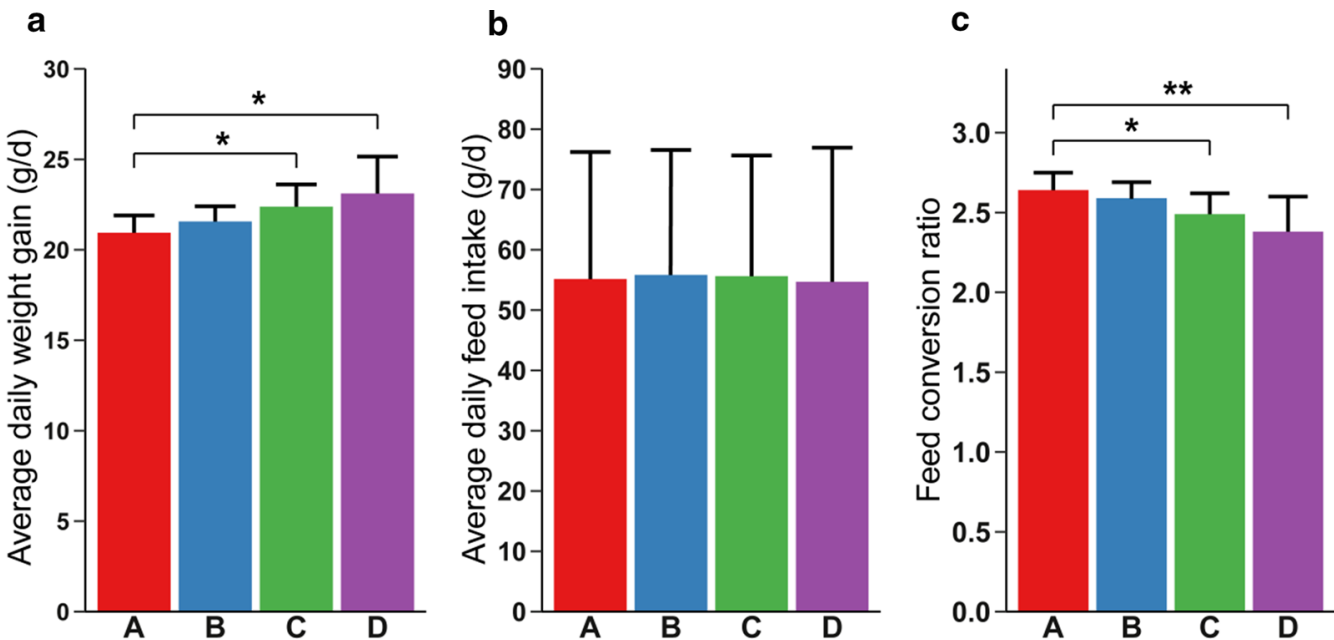

Fig. 1 Effects of different levels of purslane on growth performance of broilers. A, B, C and D correspond to the four treatment groups with $0 \%, 1 \%$, $2 \%$ and $3 \%$ purslane, respectively. Average daily weight gain (a), average daily feed intake (b) and feed conversion ratio (c) were compared among the four treatment groups. Asterisk indicates significantly changed $(p<0.05)$, double asterisk indicates extremely significantly changed ( $p<0.01)$

$2.17 \mathrm{~g}$, respectively. Subsequently, the analysis results of FCR suggested that it showed a gradual decrease trend with the increase of purslane. Among them, the FCR of the $\mathrm{C}$ and $\mathrm{D}$ groups decreased significantly, decreased by $0.15 \%$ and $0.26 \%$, respectively, especially in group $\mathrm{D}$, the decline was extremely significant $(\mathrm{p}<0.01)$. These results indicate that the addition of purslane to feed broiler chickens has no significant effect on feed intake, but gains weight significantly, achieves the purpose of improving growth performance and reducing FCR. In the meantime, our results demonstrate that the best effect of reducing the FCR was $3 \%$ purslane-fed.

\section{Assessment of sequencing data quality and OTU analysis of fecal samples}

A total of 937,475 raw tags were obtained from 12 fecal samples. After filtering a series of low-quality raw tags and removing the chimera sequences, 837,028 effective tags were obtained for subsequent analysis, and the average number of high-quality sequences for each sample was 69,752 . The length distribution range of effective tags was $350-450 \mathrm{bp}$, and the average length is $423 \mathrm{bp}$. The average GC content accounts for $52.48 \%$, and the average Q20 value reaches $97.76 \%$ (Additional file 1: Table S2).

The effective tags were clustered into OTU at a similarity level of 97\%, and OTU representative sequences were annotated. The Venn diagram (Fig. 2) was used to show the number of shared and unique OTUs among the samples after clustering. A total of 671 OTUs were obtained, and 321 OTUs were shared by the four groups. Among them, the number of unique OTUs in group A was 28, group B was 9, group C was 4, and group D was 2 .

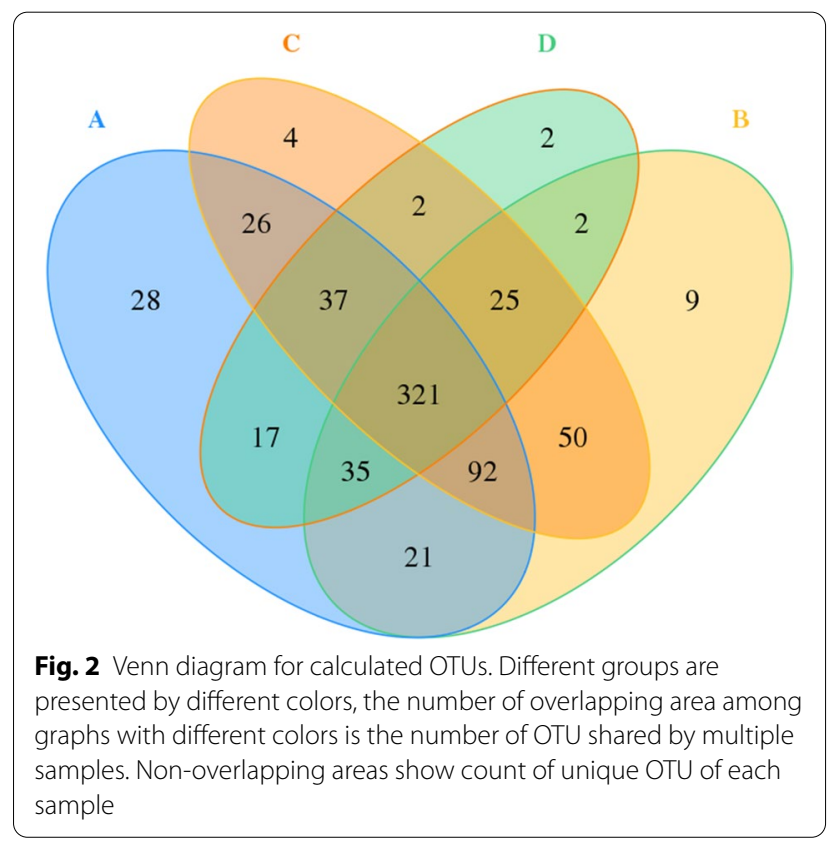

\section{Estimation of gut microbial diversity}

To verify whether the sequencing amount is sufficient for studying the microbial community, the randomly picked sequencing amount and the corresponding species number were used to construct rarefaction curve. The results revealed that all of them became relatively flat as the number of sequences analyzed increased, which indicated that the amount of sequencing was accurate and reasonable, and the depth of sequencing was enough to meet the experimental requirements (Additional file 1: 
Fig. S1a). To further confirm the reliability, the Shannon index curve was made according to the Shannon indexes of sequencing amount under different sequencing depths, which became flat with the number of sequences analyzed increased as well (Additional file 1: Fig. S1b). These results indicated that the majority of microorganisms in the samples had been captured, and the quality of the sequencing data was qualified for further analysis.
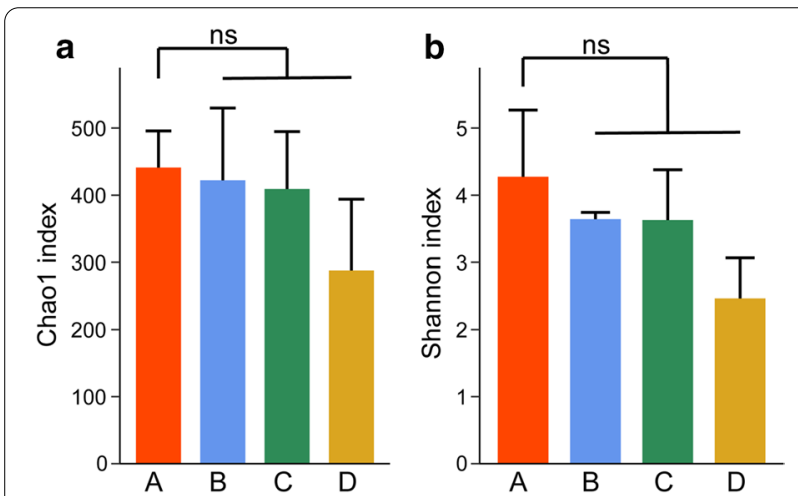

Fig. 3 Alpha diversity metrics of fecal bacterial communities. a Histogram for comparison of species richness (Chaol index) between the four groups. $\mathbf{b}$ Histogram for comparison of species diversity (Shannon index). (ns: not significant)
Subsequently, the Alpha diversity of microbial communities in the four groups of samples was measured based on the Chao1 estimators and the Shannon indexes, which represent species abundance and diversity, respectively. Compared with control group A, no significant differences were found in the Chaol and Shannon indexes of the other three groups (Fig. 3). This result shows that our groups had no effect on the abundance and diversity of the microbial communities, however, they all declined gradually, which means that when purslane was increased to a certain amount, it may have an effect on the abundance and diversity of the gut microbiota.

\section{Microbial community composition and relative abundance}

The statistical results of OTUs indicated the relative abundance of bacteria at the taxonomic level of phylum, class, order, family, and genus. The relative abundance of species on family and genus level is shown in Fig. 4. The results showed that the bacterial composition and relative abundance of the four groups were different.

At the family level (Fig. 4a), the most predominant family in the control group A was Pasteurellaceae, followed by Bacteroidaceae and Enterobacteriaceae, while the other three groups were dominated by Lactobacillaceae, followed by Pasteurellaceae or Bacteroidaceae. Moreover, it is worth paying attention that the relative abundance of

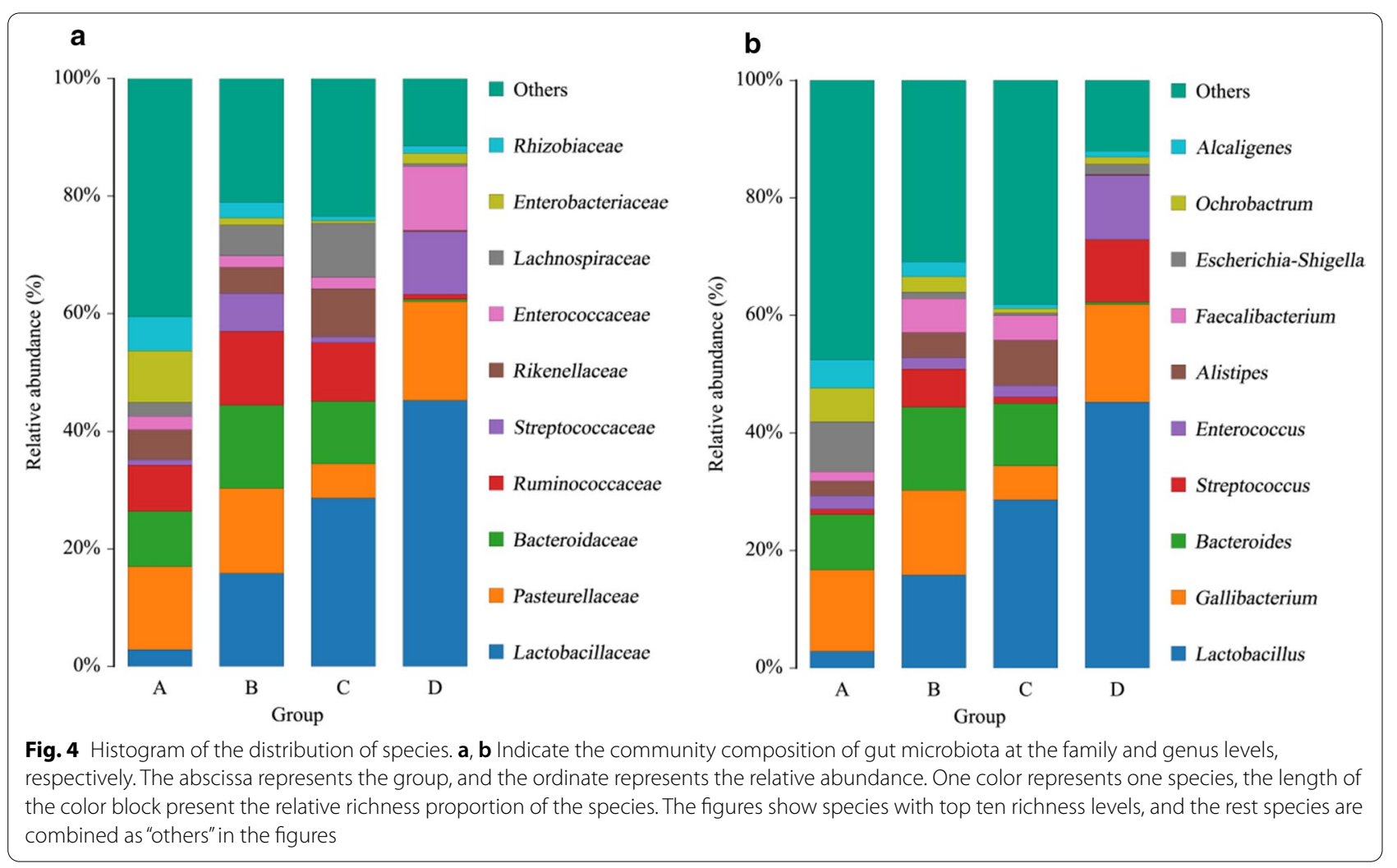


Lactobacillaceae in groups B, C and D containing purslane gradually increased with the increase of purslane, while Enterobacteriaceae rapidly decreased. Another interesting point was found that Ruminococcaceae and Lachnospiraceae had higher relative abundance in group $\mathrm{B}$ and $\mathrm{C}$, but group $\mathrm{D}$ did not. In group $\mathrm{D}$, the relative abundance of Pasteurellaceae, Enterococcaceae and Streptococcaceae were higher except for Lactobacillaceae, while the others were significantly lower.

The observation at the genus level was further implemented (Fig. 4b), and we found that the Lactobacillus displayed significant difference among the four groups of samples. Its relative abundance was only accounted for $2.85 \%$ in group A, but it accounted for $15.83 \%, 28.64 \%$ and $45.28 \%$ in groups $\mathrm{B}, \mathrm{C}$ and $\mathrm{D}$, respectively. In addition, in the control group A, the main microbial communities were Gallibacterium (13.87\%), Bacteroides (9.40\%) and Escherichia-Shigella (8.48\%). Remarkably, after purslane was fed, Lactobacillus became the most predominant genus, Gallibacterium did not change significantly, Bacteroides was significantly reduced in group D and replaced by Streptococcus and Enterococcus. Moreover, the Escherichia-Shigella enriched in group A only accounted for $1.08 \%, 0.40 \%$ and $1.68 \%$ in groups $\mathrm{B}, \mathrm{C}$ and $\mathrm{D}$. For the remaining genera, it did not indicate significant differences in abundance compared with the control.

Taken together, the addition of purslane to feed broilers could interfere with the composition of the gut microbiota, which was mainly reflected in the effect on Lactobacillus of Lactobacillaceae. On the one hand, the increase in purslane led to an increase in the relative abundance of Lactobacillus. On the other hand, the abundance of Escherichia-Shigella in Enterobacteriaceae decreased rapidly with the increase in purslane.

\section{Overview of LEfSe analysis and function prediction of gut microbiota}

In order to find out which bacteria caused the statistical differences in the microbial communities between different groups, LEfSe analysis was used to identify biomarkers. According to the established Biomarker screening criteria (LDA score $>4$ ), a total of five eligible species were identified (Fig. 5). As shown in Fig. 5a, the species with significant differences in group B was Bacteroides caecigallinarum, group $\mathrm{C}$ was uncultured_bacterium_f Lachnospiraceae, and group D was Lactobacillales and Firmicutes, which had great impact. The evolutionary branch graph of LEfSe analysis was depicted in Fig. 5b. The Cladogram shows that there were four differential bacterial taxa after added purslane. Specifically, group B had 1 species with significant difference, group $C$ had 1 genus and 1 species with significant difference and both belonged to Lachnospiraceae, and group D had 1 phylum and 1 order with significant difference.

To infer the functional changes of the gut microbiota after added purslane, we used PICRUSt. Based on the KEGG database, a total of six biological metabolic pathways were revealed at level 1 pathway, included metabolism, environmental information processing, genetic information processing, cellular processes, human diseases and organismal systems. Meanwhile, the predicted secondary pathway was found to be mainly composed of 45 sub-functions, included Global and overview maps, carbohydrate metabolism, amino acid metabolism, membrane transport, metabolism of cofactors and vitamins, energy metabolism, nucleotide metabolism, translation, replication and repair and signal transport, etc. (Additional file 2: Table S3). Furthermore, the predicted KEGG Pathways differentially abundant between four groups, after purslane was fed, carbohydrate metabolism and nucleotide metabolism were enriched, and amino acid metabolism, metabolism of cofactors and vitamins, and energy metabolism were reduced. In addition, all subfunctions in the genetic information processing had a certain increase, while some had decreased in human diseases.

\section{Discussion}

In the past few years, purslane has been widely used in various animal diets to improve animal growth and metabolism and disease resistance due to its high amounts of bioactive compounds. Abdel-Razek et al. (2019) showed that feeding purslane to Nile tilapia significantly increased the survival rate, and found plasmatic superoxide dismutase (SOD), catalase (CAT) and glutathione peroxidase (GPX) activities increased significantly with increase purslane levels as well. Zidan et al. (2014) showed that purslane effectively reduces the contents of serum total cholesterol (TC), triacylglycerols (TG) and low-density lipoproteins (LDL) accompanied with increases in high-density lipoproteins (HDL) in rats fed enriched-cholesterol diet, which contributes to antiatherosclerosis. Beyond these, many studies on purslane in chickens have been widely reported. Aydin and Dogan (2010) indicated that adding purslane to the diet of laying hens significantly increased egg production and egg weight. Sadeghi et al. (2016) indicated that purslane significantly improved the feed efficiency and antioxidant status of broilers.

Gut microbiota plays a vital role for the growth and development of animals. Diet is a source of the composition of the gut microbiota. Dietary has profound effects on microbial composition, which in turn affects a series of metabolic, hormonal and neurological processes (Frame et al. 2020). Therefore, it is necessary to modulate 
a

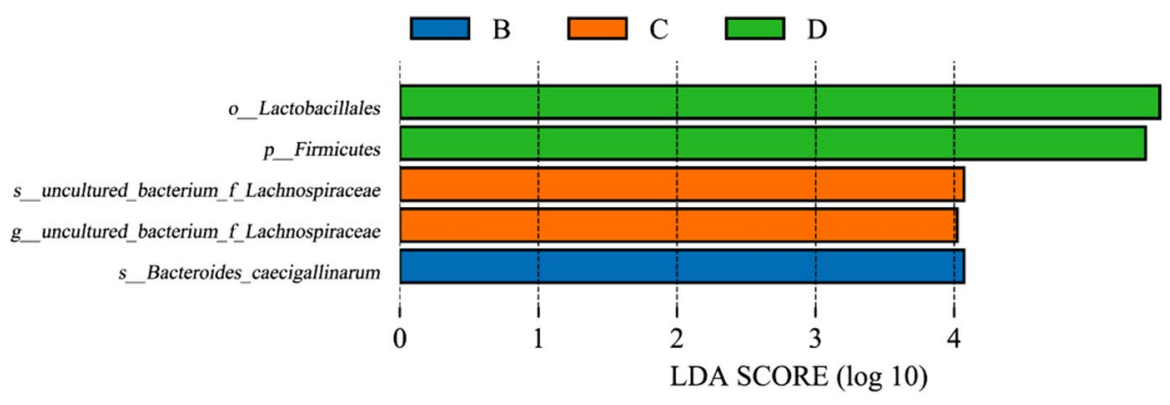

b

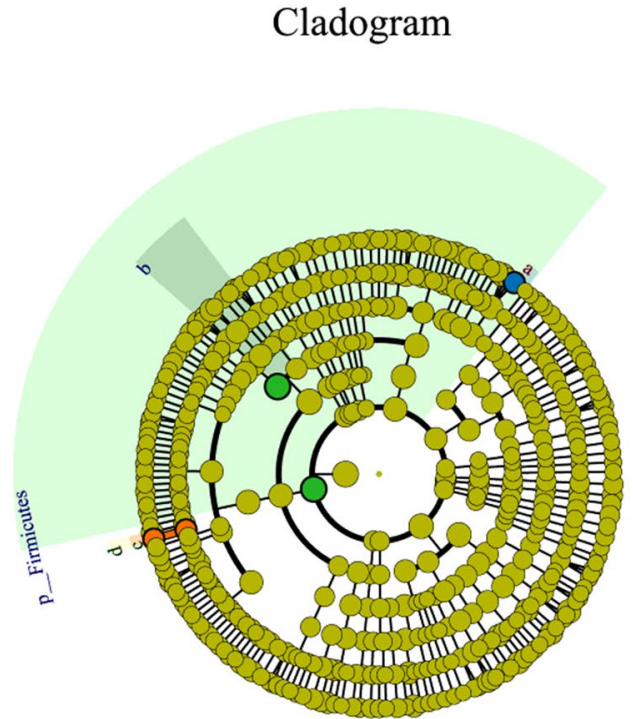

Cladogram

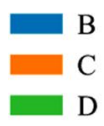

Fig. 5 The differential taxa of gut microbiota after added purslane. a LDA value distribution histogram. $\mathbf{b}$ Cladogram of LEfSe analysis. The circle radiating from inside to outside of the cladogram represent the classification levels from phylum to species. Each small circle at different classification levels represents a classification at that level, and the diameter of the circle is proportional to the relative abundance. The blue, orange, and green circles represent the bacteria enriched in groups B, C and D, respectively, whereas the yellow circles represent the taxa with no significant difference

the gut microbiota and promote its growth and development through the improvement of animal diets. Our study found that adding purslane to the diet has a significant effect on regulating the gut microbiota of broilers and enhancing their growth performance. Specifically, the body weight of broiler chickens increased with the increase of purslane. At $2 \%$ and above, the weight gain was significantly higher, whereas the FCR was lower. In the investigation of the gut microbiota, it was found that the abundance and diversity of gut microbiota were slightly reduced, but there was no significant difference, which means that $3 \%$ purslane may be the optimal content to enhance the growth performance of broilers under the premise of that the diversity of the gut microbiota is not reduced significantly.

Taking into account the difference in the amounts of purslane added, we compared the microbial communities of each group at each taxonomic level. The compositions of microbial communities are the main factors for the difference in growth performance. Our analysis showed that Lactobacillus of Lactobacillaceae was primary taxa after supplementation of diet with purslane, and accounted for a greater proportion than other differentially abundant taxa. This genus was one of the probiotics for humans and animals, which was of great significance to intestinal health. Some of the probiotics could increase the nutritional value of feed and were highly related to the animal growth performance, as well as the best alternatives to replacing antibiotics in the feed industry (Neveling and Dicks 2020). The probiotic Lactobacillus species might promote the maturation of the intestinal microbiota and enhancement of intestinal immunity against certain pathogens (Gao et al. 2017; Hardy et al. 2013). Additionally, Lactobacillus suggests a prominent role in improving chicken feed efficiency (Yan et al. 2017). Lactobacillus has been reported to lead to obesity or weight 
gain (Angelakis and Raoult 2010; Million et al. 2012). The results we obtained were in agreement with those reported, which also shows that it could promote nutrient absorption and energy extraction in the host.

Notably, the relative abundance of Lactobacillus was significantly enriched. In contrast, the abundance of Escherichia-Shigella in the Enterobacteriaceae was rapidly decreasing, which was also consistent with some studies (Sadeghi et al. 2016; Zhao et al. 2013). The vast majority of this genus are highly adapted pathogens, including species like Enterotoxigenic Escherichia coli that have been reported to cause diarrhea in piglets (Bin et al. 2018), and Shigella is a key pathogen causing bacillary dysentery (Phalipon and Sansonetti 2007). Interestingly, in the past, purslane was used to treat bacillary dysentery in China, and modern pharmacological studies have shown that purslane has a significant antibacterial effect on enteropathogenic pathogens, which may be due to the presence of a large number of flavonoids in purslane (Desta and Cherie 2018; Lei et al. 2015; Nayaka et al. 2014).

The species with the most significant differences in each group were identified through LEfSe analysis, and the results showed that Lactobacillales, Firmicutes, Lachnospiraceae and Bacteroides_caecigallinarum had a higher degree of influence. The Lactobacillales represents a morphologically, functionally and metabolically diverse group of bacteria, and lactic acid bacteria are the core of this group. This type of bacteria mediates most of the protective effects, such as anti-inflammatory by regulating the content of intestinal interleukin factor (Chih Tsai and Chou 2016; Mu et al. 2017). Obesity is associated with Firmicutes, a study suggested that the accumulation of fat was related to the increase in the abundance ratio of Firmicutes/Bacteroidetes (Turnbaugh et al. 2006). The significantly enriched taxa in this study belonged to the phylum of Firmicutes, which caused a significant increase, while the Bacteroidetes did not change much. The increase in the ratio of the two eventually led to weight gain in broilers. Meanwhile, the abundance of Bacteroidetes was found to be significantly reduced in the high-content purslane (3\%) group, which is why it has a more prominent effect on weight gain. Nevertheless, the bacteria within the Lachnospiraceae in Firmicutes had been reported to be potentially related to obesity as well as overweight (Kameyama and Itoh 2014; Tun et al. 2018). Lachnospiraceae, a family of obligate anaerobic bacteria, is involved in the production of short-chain fatty acids and the metabolism of bile acids to facilitate colonization resistance against intestinal pathogens, thereby maintaining the health of the host (Sorbara et al. 2020). The composition of the gut microbiota is closely linked to the intake of Omega-3 polyunsaturated fatty acids, particularly for bacteria of the Lachnospiraceae family (Costantini et al. 2017; Menni et al. 2017). At present, the role of Bacteroides_caecigallinarum has not been reported, and whether it affects obesity or weight gain remains poorly understood. Moreover, our data indicates that the weight gain of this group is not significant, which may be the result of low changes in the intestinal microbiota due to the low content of purslane.

The ability to convert food into body weight is of interest to the animal production industries, and the gut microbiota play an important role in the efficiency of energy extraction from diets and host metabolism (Stanley et al. 2012, 2013). PICRUSt analysis revealed changes in metabolism caused by these bacteria, suggesting that the carbohydrate metabolism pathway is the primary factor that promotes growth. Accumulating evidence indicates that Lactobacillus could improve host physiology and metabolism and has significant effects on weight modification (Drissi et al. 2017; Mountzouris et al. 2007). The bacteria of the genus Lactobacillus possess numerous carbohydrate transport systems to regulate carbon metabolism (Monedero et al. 2007). Carbohydrates are the main component and main energy supply substance of the structure of life cells. Abundant carbohydrate metabolism indicates stronger carbon source transportation or metabolism. The existence of Lactobacillus contributes to the expression of glycogen metabolism genes and glycogen accumulation, thereby increasing the capacity of harvesting energy from the diet, thus promoting more efficient absorption of calories and subsequent weight gain (Goh and Klaenhammer 2014).

In this study, the increase in relative abundance of Lactobacillus and the decrease in Escherichia-Shigella may be the most critical reasons for weight gain. Certain bacteria of these genera such as Lactobacillus paracasei and Escherichia coli could increase intracellular fat storage and enhance lipid catabolism, respectively (Tazi et al. 2018). It means that the increase Lactobacillus contributes to store fat, and the decrease of Escherichia inhibits lipids secretion, which is ultimately reflected in the body weight gain. For other taxa, such as Ruminococcaceae and Lachnospiraceae, they have also been found to contain key carbohydrate active enzymes, sugar transport mechanisms and metabolic pathways, so that they are more specialized for the degradation of complex plant material (Biddle et al. 2013), which may be one of the reasons for the significant enrichment in animal intestines after feeding purslane. In our study, the weight gain of the $\mathrm{C}(2 \%)$ and D (3\%) groups was significant, and they were consistent in terms of the increase in the relative abundance of beneficial bacteria and the decrease in pathogenic bacteria. These findings, in accordance with the previous reports that gut microbiota is improved by 
purslane, might contribute to concluding that the effects of purslane on weight gain might depend on its modulation effect on gut microbiota.

In conclusions, this study demonstrated that the intake of $2 \%$ and $3 \%$ purslane could significantly increase the body weight gain and lead to a reduction in FCR. Moreover, purslane-fed increased the relative abundance of beneficial bacteria in the chicken intestines. What's more, purslane-fed drastically modified the gut microbial species at the family level, increasing the gut microbiota associated with obesity or weight gain, such as Lactobacillaceae and its genus Lactobacillus. Meanwhile, purslane-fed decreased the relative abundance of harmful bacteria that has been reported to be related to diseases, such as Escherichia-Shigella in family Enterobacteriaceae. Our findings indicated that the potential applications of purslane in the body weight gain might closely depend on its modulatory effect on gut microbiota. In short, adding appropriate purslane to broiler diets could increase the abundance of Lactobacillus in the gut microbiota and promote growth performance, thereby producing safer and healthier livestock products.

\section{Supplementary Information}

The online version contains supplementary material available at https://doi. org/10.1186/s13568-021-01190-z.

Additional file 1: Table S1. Composition and nutrient levels of basal diets for broilers. Table S2. Statistics of sample sequencing data processing result. Figure S1. Rarefaction curve and Shannon index curve drawn based on sequencing data.

Additional file 2: Table S3. Predicted KEGG Pathways differentially abundant between four groups.

\section{Acknowledgements}

We thank Mr. Fangxing Bao, Mr. Guangwen Zeng and Mr. Xiujian Zhong for technical assistance and sample collection in the poultry breeding process. We also thank Dr. Hebin for his assistance in preparing the manuscript.

\section{Authors' contributions}

All authors have contributed to this study. All authors read and approved the final manuscript.

\section{Funding}

This work was funded supported by the National Natural Science Foundation of China (Grant No. 31760625); Science and Technology Project of Education Department of Jiangxi Province (Grant Nos. GJJ190581, GJJ190594); the earmarked fund for Innovation Project of Graduate Students in Jiangxi Provincial Education Department (Jiangxi Science \& Technology Normal University) (Grant No. YC2020-S565).

\section{Availability of data and materials}

All raw sequences were submitted to the NCBI Sequence Read Archive (https ://www.ncbi.nlm.nih.gov/sra/) under BioProject PRJNA670355. The data supporting the conclusion of this article are included in this article.

\section{Ethics approval and consent to participate}

The Animal experiments were conducted in accordance with the guidelines established and approved by the Animal Management Committee of Jiangxi Science \& Technology Normal University.
Consent for publication

Not applicable.

\section{Competing interests}

The authors declare that they have no competing interests.

\section{Author details}

1 School of life science, Jiangxi Science and Technology Normal University, 330013 Nanchang, China. ${ }^{2}$ Jiangxi Key Laboratory of Bioprocess Engineering, Jiangxi Science and Technology Normal University, 330013 Nanchang, China.

3 Jiangxi Yifeng County Qiaoxi Veterinary Station, 336300 Yifeng, Jiangxi, China.

Received: 28 September 2020 Accepted: 8 February 2021

Published online: 23 February 2021

\section{References}

Abdallah A, Zhang P, Zhong Q, Sun Z (2019) Application of Traditional Chinese Herbal Medicine By-products as Dietary Feed Supplements and Antibiotic Replacements in Animal Production. Curr Drug Metab 20(1):54-64. https://doi.org/10.2174/1389200219666180523102920

Abdel-Razek N, Awad SM, Abdel-Tawwab M (2019) Effect of dietary purslane (Portulaca oleracea L.) leaves powder on growth, immunostimulation, and protection of Nile tilapia, Oreochromis niloticus against Aeromonas hydrophila infection. Fish Physiol Biochem 45(6):1907-1917. https://doi. org/10.1007/s10695-019-00685-8

Angelakis E, Raoult D (2010) The increase of Lactobacillus species in the gut flora of newborn broiler chicks and ducks is associated with weight gain. PLoS One 5(5):e10463. https://doi.org/10.1371/journal.pone.0010463

Angelakis E (2017) Weight gain by gut microbiota manipulation in productive animals. Microb Pathog 106:162-170. https://doi.org/10.1016/j.micpa th.2016.11.002

Aydin R, Dogan I (2010) Fatty acid profile and cholesterol content of egg yolk from chickens fed diets supplemented with purslane (Portulaca oleracea L.). J Sci Food Agric 90(10):1759-1763. https://doi.org/10.1002/jsfa.4018

Biddle A, Stewart L, Blanchard J, Leschine S (2013) Untangling the genetic basis of fibrolytic specialization by Lachnospiraceae and Ruminococcaceae in diverse gut communities. Diversity 5(3):627-640. https://doi. org/10.3390/d5030627

Bin P, Tang Z, Liu S, Chen S, Xia Y, Liu J, Wu H, Zhu G (2018) Intestinal microbiota mediates Enterotoxigenic Escherichia coli-induced diarrhea in piglets. BMC Vet Res 14(1):385. https://doi.org/10.1186/s12917-018-1704-9

Bokulich NA, Subramanian S, Faith JJ, Gevers D, Gordon Jl, Knight R, Mills DA, Caporaso JG (2013) Quality-filtering vastly improves diversity estimates from Illumina amplicon sequencing. Nat Methods 10(1):57-59. https:// doi.org/10.1038/nmeth.2276

Bolger AM, Lohse M, Usadel B (2014) Trimmomatic: a flexible trimmer for Illumina sequence data. Bioinformatics30(15):2114-2120. https://doi. org/10.1093/bioinformatics/btu170

Caporaso JG, Kuczynski J, Stombaugh J, Bittinger K, Bushman FD, Costello EK, Fierer N, Pena AG, Goodrich JK, Gordon Jl, Huttley GA, Kelley ST, Knights D, Koenig JE, Ley RE, Lozupone CA, McDonald D, Muegge BD, Pirrung M, Reeder J, Sevinsky JR, Turnbaugh PJ, Walters WA, Widmann J, Yatsunenko T, Zaneveld J, Knight R (2010) QIIME allows analysis of high-throughput community sequencing data. Nat Methods 7(5):335-336. https://doi. org/10.1038/nmeth.f.303

Chao A, Chazdon RL, Colwell RK, Shen TJ (2006) Abundance-based similarity indices and their estimation when there are unseen species in samples. Biometrics 62(2):361-371. https://doi.org/10.111 1/j.1541-0420.2005.00489.x

Chih Tsai C, Chou LC (2016) An in vitro investigation of the antagonistic effects of multiple strains of Lactobacillales on Salmonella enterica Serovar Choleraesuis. Appli Micro Open Access 2:1. https://doi.org/10.4172/24719315.1000109

Costantini L, Molinari R, Farinon B, Merendino N (2017) Impact of omega-3 fatty acids on the gut microbiota. Int J Mol Sci 18(12):2645. https://doi. org/10.3390/ijms18122645 
Dabke K, Hendrick G, Devkota S (2019) The gut microbiome and metabolic syndrome. J Clin Invest 129(10):4050-4057. https://doi.org/10.1172/JCl12 9194

Desta ZY, Cherie DA (2018) Determination of antioxidant and antimicrobial activities of the extracts of aerial parts of Portulaca quadrifida. Chem Cent J 12(1):146. https://doi.org/10.1186/s13065-018-0514-2

Drissi F, Raoult D, Merhej V (2017) Metabolic role of lactobacilli in weight modification in humans and animals. Microb Pathog 106:182-194. https://doi. org/10.1016/j.micpath.2016.03.006

Edgar RC, Haas BJ, Clemente JC, Quince C, Knight R (2011) UCHIME improves sensitivity and speed of chimera detection. Bioinformatics 27(16):21942200. https://doi.org/10.1093/bioinformatics/btr381

Edgar RC (2013) UPARSE: highly accurate OTU sequences from microbial amplicon reads. Nat Methods 10(10):996-998. https://doi.org/10.1038/ nmeth.2604

Frame LA, Costa E, Jackson SA (2020) Current Explorations of Nutrition and the Gut Microbiome: A Comprehensive Evaluation of the Review Literature. Nutr Rev 78(10):798-812. https://doi.org/10.1093/nutrit/nuz106

Gao P, Ma C, Sun Z, Wang L, Huang S, Su X, Xu J, Zhang H (2017) Feed-additive probiotics accelerate yet antibiotics delay intestinal microbiota maturation in broiler chicken. Microbiome 5(1):91. https://doi.org/10.1186/s4016 8-017-0315-1

Goh YJ, Klaenhammer TR (2014) Insights into glycogen metabolism in Lactobacillus acidophilus: impact on carbohydrate metabolism, stress tolerance and gut retention. Microb Cell Fact 13:94. https://doi.org/10.1186/s1293 4-014-0094-3

Habibian M, Sadeghi G, Karimi A (2019) Comparative effects of powder aqueous and methanolic extracts of purslane (Portulaca oleracea L.) on growth performance, antioxidant status, abdominal fat deposition and plasma lipids in broiler chickens. Anim Prod Sci 59(1):89-100. https://doi. org/10.1071/AN17352

Hardy H, Harris J, Lyon E, Beal J, Foey AD (2013) Probiotics, prebiotics and immunomodulation of gut mucosal defences: homeostasis and immunopathology. Nutrients 5(6):1869-1912. https://doi.org/10.3390/nu506 1869

Huson DH, Auch AF, Qi J, Schuster SC (2007) MEGAN analysis of metagenomic data. Genome Res 17(3):377-386. https://doi.org/10.1101/gr.5969107

Kameyama K, Itoh K (2014) Intestinal colonization by a Lachnospiraceae bacterium contributes to the development of diabetes in obese mice. Microbes Environ 29(4):427-430. https://doi.org/10.1264/jsme2.ME14054

Kartikasari LR, Hertanto BS, Nuhriawangsa AMP (2018) Carcass and cut yields of broiler chickens fed diet containing purslane meal rich in omega-3 fats. Iop C Ser Earth Env 102:012088. https://doi.org/10.1088/17551315/102/1/012088

Lei X, Li J, Liu B, Zhang N, Liu H (2015) Separation and Identification of Four New Compounds with Antibacterial Activity from Portulaca oleracea L. Molecules 20(9):16375-16387. https://doi.org/10.3390/molecules200916 375

Magoc T, Salzberg SL (2011) FLASH: fast length adjustment of short reads to improve genome assemblies. Bioinformatics 27(21):2957-2963. https:// doi.org/10.1093/bioinformatics/btr507

Menni C, Zierer J, Pallister T, Jackson MA, Long T, Mohney RP, Steves CJ, Spector TD, Valdes AM (2017) Omega-3 fatty acids correlate with gut microbiome diversity and production of $\mathrm{N}$-carbamylglutamate in middle aged and elderly women. Sci Rep 7(1):11079. https://doi.org/10.1038/s41598-01710382-2

Million M, Angelakis E, Paul M, Armougom F, Leibovici L, Raoult D (2012) Comparative meta-analysis of the effect of Lactobacillus species on weight gain in humans and animals. Microb Pathog 53(2):100-108. https://doi. org/10.1016/j.micpath.2012.05.007

Monedero V, Maze A, Boel G, Zuniga M, Beaufils S, Hartke A, Deutscher J (2007) The phosphotransferase system of Lactobacillus casei: regulation of carbon metabolism and connection to cold shock response. J Mol Microbiol Biotechnol 12(1-2):20-32. https://doi.org/10.1159/000096456

Mountzouris KC, Tsirtsikos P, Kalamara E, Nitsch S, Schatzmayr G, Fegeros K (2007) Evaluation of the efficacy of a probiotic containing Lactobacillus, Bifidobacterium, Enterococcus, and Pediococcus strains in promoting broiler performance and modulating cecal microflora composition and metabolic activities. Poult Sci 86(2):309-317. https://doi.org/10.1093/ ps/86.2.309
Mu Q, Zhang H, Liao X, Lin K, Liu H, Edwards MR, Ahmed SA, Yuan R, Li L, CecereTE, Branson DB, Kirby JL, Goswami P, Leeth CM, Read KA, Oestreich KJ, Vieson MD, Reilly CM, Luo XM (2017) Control of lupus nephritis by changes of gut microbiota. Microbiome 5(1):73. https://doi.org/10.1186/ s40168-017-0300-8

Nayaka HB, Londonkar RL, Umesh MK, Tukappa A (2014) Antibacterial attributes of Apigenin, isolated from Portulaca oleracea L. Int J Bacteriol 2014:175851. https://doi.org/10.1155/2014/175851

Neveling DP, Dicks LMT (2020) Probiotics: an antibiotic replacement strategy for healthy broilers and productive rearing. Probiotics Antimicrob Proteins. https://doi.org/10.1007/s12602-020-09640-z

Ondov BD, Bergman NH, Phillippy AM (2011) Interactive metagenomic visualization in a Web browser. BMC Bioinform 12:385. https://doi. org/10.1186/1471-2105-12-385

Parks DH, Tyson GW, Hugenholtz P, Beiko RG (2014) STAMP: statistical analysis of taxonomic and functional profiles. Bioinformatics 30(21):3123-3124. https://doi.org/10.1093/bioinformatics/btu494

Phalipon A, Sansonetti PJ (2007) Shigella's ways of manipulating the host intestinal innate and adaptive immune system: a tool box for survival? Immunol Cell Biol 85(2):119-129. https://doi.org/10.1038/sj.icb7100025

Quast C, Pruesse E, Yilmaz P, Gerken J, Schweer T, Yarza P, Peplies J, Glockner FO (2013) The SILVA ribosomal RNA gene database project: improved data processing and web-based tools. Nucleic Acids Res 41 (Database issue):D590-D596. https://doi.org/10.1093/nar/gks1219

Rahimi VB, Ajam F, Rakhshandeh H, Askari VR (2019) A pharmacological review on Portulaca oleracea L.: focusing on anti-inflammatory, anti- oxidant, immuno-modulatory and antitumor activities. J Pharmacopuncture 22(1):7-15. https://doi.org/10.3831/KPI.2019.22.001

Sadeghi G, Karimi A, Shafeie F, Vaziry A, Farhadi D (2016) The effects of purslane (Portulaca oleracea L.) powder on growth performance, carcass characteristics, antioxidant status, and blood metabolites in broiler chickens. Livest Sci 184:35-40. https://doi.org/10.1016/j.livsci.2015.12.003

Schloss PD, Westcott SL, Ryabin T, Hall JR, Hartmann M, Hollister EB, Lesniewski RA, Oakley BB, Parks DH, Robinson CJ, Sahl JW, Stres B, Thallinger GG, Van Horn DJ, Weber CF (2009) Introducing mothur: open-source, platformindependent, community-supported software for describing and comparing microbial communities. Appl Environ Microbiol 75(23):7537-7541. https://doi.org/10.1128/AEM.01541-09

Schunck WH, Konkel A, Fischer R, Weylandt KH (2018) Therapeutic potential of omega-3 fatty acid-derived epoxyeicosanoids in cardiovascular and inflammatory diseases. Pharmacol Ther 183:177-204. https://doi. org/10.1016/j.pharmthera.2017.10.016

Segata N, Izard J, Waldron L, Gevers D, Miropolsky L, Garrett WS, Huttenhower C (2011) Metagenomic biomarker discovery and explanation. Genome Biol 12(6):R60. https://doi.org/10.1186/gb-2011-12-6-r60

Shahidi F, Ambigaipalan P (2018) Omega-3 polyunsaturated fatty acids and their health benefits. Annu Rev Food Sci Technol 9:345-381. https://doi. org/10.1146/annurev-food-111317-095850

Sorbara MT, Littmann ER, Fontana E, Moody TU, Kohout CE, Gjonbalaj M, Eaton V, Seok R, Leiner IM, Pamer EG (2020) Functional and genomic variation between human-derived isolates of Lachnospiraceae reveals inter-and intra-species diversity. Cell Host Microbe 28(1):134-146.e4. https://doi. org/10.1016/j.chom.2020.05.005

Stanley D, Denman SE, Hughes RJ, Geier MS, Crowley TM, Chen H, Haring VR, Moore RJ (2012) Intestinal microbiota associated with differential feed conversion efficiency in chickens. Appl Microbiol Biotechnol 96(5):13611369. https://doi.org/10.1007/s00253-011-3847-5

Stanley D, Geier MS, Denman SE, Haring VR, Crowley TM, Hughes RJ, Moore RJ (2013) Identification of chicken intestinal microbiota correlated with the efficiency of energy extraction from feed. Vet Microbiol 164(1-2):8592.https://doi.org/10.1016/j.vetmic.2013.01.030

Tazi A, Araujo JR, Mulet C, Arena ET, Nigro G, Pedron T, Sansonetti PJ (2018) Disentangling host-microbiota regulation of lipid secretion by enterocytes: insights from commensals Lactobacillus paracasei and Escherichia coli. mBio 9(5):e01493-e01418. https://doi.org/10.1128/mBio.01493-18

Tun HM, Bridgman SL, Chari R, Field CJ, Guttman DS, Becker AB, Mandhane PJ, Turvey SE, Subbarao P, Sears MR, Scott JA, Kozyrskyj AL, Canadian Healthy Infant Longitudinal Development Study I (2018) Roles of birth mode and infant gut microbiota in intergenerational transmission of overweight and obesity from mother to offspring. JAMA Pediatr 172(4):368-377. https://doi.org/10.1001/jamapediatrics.2017.5535 
Turnbaugh PJ, Ley RE, Mahowald MA, Magrini V, Mardis ER, Gordon JI (2006) An obesity-associated gut microbiome with increased capacity for energy harvest. Nature 444(7122):1027-1031. https://doi.org/10.1038/natur e05414

Uddin MK, Juraimi AS, Hossain MS, Nahar MA, Ali ME, Rahman MM (2014) Purslane weed (Portulaca oleracea): a prospective plant source of nutrition omega-3 fatty acid, and antioxidant attributes. ScientificWorldJournal 2014:951019. https://doi.org/10.1155/2014/951019

Wang Q, Garrity GM, Tiedje JM, Cole JR (2007) Naive Bayesian classifier for rapid assignment of rRNA sequences into the new bacterial taxonomy. Appl Environ Microbiol 73(16):5261-5267. https://doi.org/10.1128/AEM.00062 $-07$

Yan W, Sun C, Yuan J, Yang N (2017) Gut metagenomic analysis reveals prominent roles of Lactobacillus and cecal microbiota in chicken feed efficiency. Sci Rep 7:45308. https://doi.org/10.1038/srep45308

Zhao XH, He X, Yang XF, Zhong XH (2013) Effect of Portulaca oleracea extracts on growth performance and microbial populations in ceca of broilers. Poult Sci 92(5):1343-1347. https://doi.org/10.3382/ps.2012-02434
Zhou YX, Xin HL, Rahman K, Wang SJ, Peng C, Zhang H (2015) Portulaca oleracea $L$.: a review of phytochemistry and pharmacological effects. Biomed Res Int 2015:925631. https://doi.org/10.1155/2015/925631

Zidan Y, Bouderbala S, Djellouli F, Lacaille-Dubois MA, Bouchenak M (2014) Portulaca oleracea reduces triglyceridemia, cholesterolemia, and improves lecithin: cholesterol acyltransferase activity in rats fed enriched-cholesterol diet. Phytomedicine 21(12):1504-1508. https://doi.org/10.1016/j. phymed.2014.07.010

\section{Publisher's note}

Springer Nature remains neutral with regard to jurisdictional claims in published maps and institutional affiliations.

\section{Submit your manuscript to a SpringerOpen ${ }^{\circ}$ journal and benefit from:}

- Convenient online submission

- Rigorous peer review

- Open access: articles freely available online

- High visibility within the field

- Retaining the copyright to your article

Submit your next manuscript at $\boldsymbol{\nabla}$ springeropen.com 